\title{
Estrutura e diversidade de uma floresta de Miombo em Taratibu, Norte de Moçambique
}

\author{
Salvador José António NANVONAMUQUITXO ${ }^{1 *}$, Fane Benedito Eduardo Duarte MACUEIA ${ }^{1}$, \\ Marcelino Inácio CARAVELA ${ }^{1}$
}

${ }^{1}$ Repartição de Botânica, Faculdade de Ciências Naturais, Universidade Lúrio, Pemba, Cabo Delgado, Moçambique.

*E-mail: agiradors@gmail.com

Recebido em setembro/2018; Aceito em setembro/2019.

RESUMO: O objetivo deste trabalho foi avaliar a estrutura e diversidade de uma floresta de Miombo localizada em Taratibu no Parque Nacional das Quirimbas região norte de Moçambique, a fim de subsidiar ações de manejo e conservação. Uma área de 3,1 ha foi dividida em 31 parcelas de 50 x $20 \mathrm{~m}$, onde foi mensurado o DAP $\geq 10,0$ $\mathrm{cm}$ das árvores observadas. A composição florística, parâmetros fitossociológicos e estrutura diamétrica foram usados para avaliar a estrutura horizontal. O índice de Shannon Wiener $\left(\mathrm{H}^{\prime}\right)$ e Pielou $\left(\mathrm{J}^{\prime}\right)$ para a diversidade. Foram registrados 1111 árvores, distribuídas em 38 espécies, 36 géneros e 12 famílias. A Fabaceae foi a família de maior importância ecológica com 39\% das espécies. A floresta apresentou uma densidade de 358 arv.ha ${ }^{-1}$ e área basal de $16,57 \mathrm{~m}^{2} \mathrm{ha}^{-1}$. A distribuição das árvores por classes diamétricas seguiu o padrão j-invertido, comum em florestas naturais, apesar disso, as classes 12,5; 17,5 e 42,5 cm apresentaram-se com deficit de 4, 10, e 3 arv.ha ${ }^{-1}$ respectivamente. $\mathrm{O} \mathrm{H}^{\prime}$ e $\mathrm{J}^{\prime}$ foram de 2,19 e 0,60 respectivamente. Taratibu apresenta estrutura e diversidade florística estável, há no entanto necessidade de intervenção de manejo de modo a garantir a conservação deste ecossistema.

Palavras-chave: Taratibu; fitossociologia; conservação florestal; Parque Nacional das Quirimbas.

\section{Structure and forest diversity of Miombo woodland in Taratibu, North of Mozambique}

\begin{abstract}
The aim of this work was to evaluate the structure and forest diversity Miombo woodland located in Taratibu, in Quirimbas National park, northern of Mozambique, in order to subsidize actions of management and conservation. 3,1 ha, were divided into 31 plots of $50 \times 20 \mathrm{~m}$, where the $\mathrm{DBH} \geq 10,0 \mathrm{~cm}$ of all observed trees were measured. Floristic composition, phytosociological parameters and diametric structure were used to evaluate the horizontal structure. Shannon Wiener and Pielou index were used to evaluate the forest diversity. There were 1111 trees, distributed in 38 species, 36 genera and 12 families. Fabaceae was the most ecologically important family with $39 \%$ of all species. The woodland had a density of 358 trees per hectare and a basal area of $16,57 \mathrm{~m}^{2} \cdot \mathrm{ha}^{-1}$. The distribution of trees on diametric structure followed the j-inverted, typical of natural forests, however, the diametric classes of 12,5; 17,5 and 42,5 cm, showed, deficit of 4, 10, and 3 trees per hectare respectively. The Shannon and Pielou index were 2,19 and 0,6 respectively. Taratibu has stabilized structure and forest diversity, however management action is needed to maintain this ecosystem conserved.
\end{abstract}

Keywords: Taratibu; phytosociology; forest conservation; Quirimbas National Park.

\section{INTRODUÇÃO}

Atualmente as florestas tropicais compreendem um ecossistema terrestre muito ameaçado de extinção, devido essencialmente a mudanças climáticas, fogo, agricultura itinerante e crescimento populacional. Além de existirem poucas áreas destinadas à conservação e à protecção das mesmas (MILES et al., 2006). Este fato tem implicações na redução da biodiversidade e declínio de importantes serviços ecossistémicos.

A floresta de Miombo cobre aproximadamente $10 \%$ dos ambientes florestais do continente africano, e constitui o bioma mais importante da região Sul e Oriental da África em termos de biodiversidade (DEWEES et al., 2010). Este bioma compreende uma extensão de 2,7 milhões de $\mathrm{km}^{2}$ de área, desde o extremo oeste no Oceano Atlântico prolongando-se até ao extremo leste, próximo ao Oceano Índico, entre os paralelos $3^{\circ} \mathrm{S}$ a $26^{\circ} \mathrm{S}$ (MALMER, 2007), sendo a principal fitofisionomia florestal de países como Angola, Botsuana, Malawi, Moçambique, Tanzânia, Zâmbia e Zimbábue (CHIDUMAYO, 2013).

Em Moçambique, Miombo e cobre 2/3 da área total, e este distribui-se um pouco por todo território, apresentando biodiversidade característica conforme as condições ecológicas de cada região (RIBEIRO et al., 2008).

O elevado índice de pobreza associado a dependências das comunidades rurais aos recursos deste bioma, pressupõe que, ao longo do tempo essas áreas sofram perturbações decorrentes de actividades antrópicas (WILLIAMS et al., 2008). Apesar disto, ainda existem áreas que apresentam um grau elevado de conservação e que são referência para pesquisas, como é o caso de Taratibu.

Taratibu é uma das áreas da região norte de Moçambique que apesar de perturbações antrópicas ocorridas ao longo de 
tempo, ainda detém boa parte do seu ecossistema conservado, garantindo abrigo para várias espécies da flora e fauna terrestre. No entanto, os vários habitats e comunidades vegetais que a compõem, ainda não foram cientificamente descritos. Por isso neste estudo busca-se uma descrição da estrutura horizontal desta floresta através de um inventário florestal como forma de prover informações fundamentais, para entender a dinâmica deste ecossistema e subsidiar estratégias que visão a sua conservação (AIMI et al., 2017).

Para Coraiola; Netto (2003) os resultados das análises da estrutura de uma floresta também permitem fazer deduções sobre a origem, características ecológicas, dinamismo e tendências do futuro desenvolvimento da mesma, elementos básicos e fundamentais para o planejamento do Manejo Silvicultural. Santos et al. (2016) afirmam que qualquer intervenção na floresta carece de informações sobre parâmetros estruturais e funcionais que regulam a dinâmica florestal.

Assim, estudos sobre a estrutura da floresta, a composição florística e dinâmica florestal, são passos importantes para subsidiar o manejo do bioma de Miombo e possibilitar o desenvolvimento de programas de conservação da diversidade em Moçambique (HOFIÇO; FLEIG, 2015; RIBEIRO et al., 2017).

Neste contexto, o presente estudo tem como objectivo avaliar a estrutura horizontal e a diversidade de uma floresta do Miombo localizada em Taratibu na região norte de Moçambique, a fim de subsidiar ações de manejo e conservação.

\section{MATERIAL E MÉTODOS}

\section{1. Área de estudo}

$\mathrm{O}$ estudo foi realizado em uma área de Taratibu, região norte de Moçambique situada entre as coordenadas $12^{\circ} 50^{\prime} \mathrm{S}$ e $12^{\circ} 38^{\prime} \mathrm{S}$ latitudes Sul, e $39^{\circ} 32^{\prime} \mathrm{E}$ e $39^{\circ} 58^{\prime} \mathrm{E}$ longitudes Leste. A área é propriedade concessionária do Sr. Jacobs Von Landsberg. (WWF, 2013). Taratibu é parte integrante do bloco A do Parque Nacional das Quirimbas, com uma área aproximada a 35000 ha.

A região é caracterizada por um clima sub-húmido seco com duas estações distintas, estação chuvosa no verão e seca no inverno. A temperatura média ao longo do ano varia entre $20{ }^{\circ} \mathrm{C}$ e $25^{\circ} \mathrm{C}$ e a precipitação média anual ente 800 a 1200 $\mathrm{mm}$, distribuídas entre os meses de Outubro a Março. A reserva é atravessada pelo rio Nculo e rio Montepuez ambos de regime periódico (MAE, 2005).

A fitoecologia típica da região é a floresta de Miombo, em estágio de sucessão primário como fisionomia predominante (GELDENHUYS, 2005; SITOE et al., 2010). Essa fitofisionomia forma um mosaico na cobertura vegetal da área de estudo relacionado às variações ambientais (umidade, temperatura e precipitação), microclima e a ocorrência de fogo (ZOLHO, 2005; RIBEIRO et al., 2008; RYAN; WILLIAMS, 2011).

A área tem histórico de perturbação por exploração madeireira, uso de fogo e a prática comum de agricultura itinerante, fatores estes observados no levantamento dos dados em campo.

\subsection{Desenho amostral e obtenção dos dados}

$\mathrm{Na}$ área, foi instalada sistematicamente uma grade amostral de 31 unidades de $1000 \mathrm{~m}^{2}(50 \mathrm{~m} \times 20 \mathrm{~m})$, distanciadas de $500 \mathrm{~m}$ entre linhas e $50 \mathrm{~m}$ entre parcelas, respectivamente, visando cobrir possíveis variações na vegetação. Todos os indivíduos lenhosos, com diâmetro a 1,30 m do solo (DAP) igual ou superior a $10,0 \mathrm{~cm}$, foram mensurados com suta, georreferenciadas com recurso a Geographic Position System (GPS) e identificados individualmente por meio de uma plaqueta para maior praticidade em possíveis remedições. Foi coletado o material botânico e a identificação das espécies foi utilizada literatura especializada (VAN WYK; VAN WYK, 2011). A nomenclatura das espécies segue o sistema de classificação de Angiosperm Phylogeny Group versão IV (APG, 2016).

\subsection{Análise de dados}

A estrutura horizontal foi avaliada por meio do cálculo dos parâmetros fitossociológicos absolutos e relativos de densidade, dominância e frequência das espécies, bem como pelo valor de importância, parâmetro resultante do somatório dos três parâmetros anteriormente citados (KENT; COKER, 2011), e a estrutura diamétrica através do histograma de frequência da distribuição das árvores em classes de diâmetro com amplitude de cinco centímetros (ISANGO, 2007; SHIRIMA et al., 2011), e posteriormente foi ajustada ao modelo exponencial negativo de Meyer a distribuição diamétrica (KERSHAW JR et al., 2016). A diversidade foi estimada pelo índice de Shannon-Wiener (H') na escala logarítmica e a equabilidade conforme Pielou (J') (KENT; COKER, 2011)

Para calcular os parâmetros fitossociológicos e a diversidade florística utilizou-se o software Mata Nativa 2 (CIENTEC, 2006) e o ajuste do modelo para descrever a distribuição diamétrica utilizou-se o software Statistical Analysis System 9.2 (SAS INSTITUTE, 2011).

\section{RESULTADOS}

3.1. Composição florística e fitossociológica

Um total de 1111 árvores foram registradas, e identificados 38 espécies de 33 géneros e 12 famílias na área de estudo. A Julbernardia paniculata (Benth.) Troupin foi a espécie ecologicamente mais importante com 63,2 arv.ha $^{-1}$ correspondente a 13,3\% do valor de importância (VI), seguido de Combretum adenogonium Steud. ex A.Rich. com 26,1 arv.ha ${ }^{-1}$ (6,1\% de VI), Pteleopsis myrtifolia Engl. \& Diels com 17,1 arv.ha $^{-1}(5,4 \%$ VI), Burkea africana Hook., com 19,7 arv.ha ${ }^{-1}(5,4 \%$ de VI) e Brachystegia spiciformis Benth. com 14,8 arv.ha $^{-1}(5,4 \%$ de VI). Estas cinco espécies representaram $35,6 \%$ do VI total da área de estudo (Tabela 1 ).

As famílias com maior número de espécies foram Fabaceae (39\%) e Combretaceae (11\%) (Figura 1). Outras dez famílias apresentaram entre $8 \%$ a 3\% das espécies da área. Estas duas principais famílias representaram metade dos espécimes inventariados, ficando as dez famílias restantes, representando a outra metade, evidenciando a baixa abundância relativa de árvores nestas famílias.

\subsection{Estrutura diamétrica}

A distribuição diamétrica da floresta pode ser observada na Figura 2, tendo-se apresentado na forma de $J$-invertido, típica de florestas naturais. A densidade total desta vegetação foi de 358 arv.ha- ${ }^{-1}$ com área basal de $16,57 \mathrm{~m}^{2} \cdot \mathrm{ha}^{-1}$. O coeficientes da equação de distribuição de árvores ajustada a função de Meyer foram $\beta_{0}=5,928, \beta_{1}=-0,087$, o coeficiente de determinação ajustado $\left(R_{a j}^{2}=0,970\right)$, erro padrão das estimativas $\left(S_{y x}=0,273\right)$ e o valor de $F$ de 362,39 , demostrando 
uma distribuição irregular de árvores, nas classes 12,5; 17,5 e $42,5 \mathrm{~cm}$, que apresentaram-se com deficit de 4, 10, e 3 arv.ha${ }^{1}$ respectivamente, típico de uma floresta não balanceada.

\subsection{Diversidade de espécies}

A diversidade florística estimada pelo índice de ShannonWiener (H') foi alta de 2,19 nats.ind ${ }^{-1}$ e equabilidade de Pielou (J') foi de 0,60 .

Tabela 1. Parâmetros fitossociológicos da floresta de Miombo, na reserva de Taratibu, norte de Moçambique.

Table 1. Phytosociological parameter of the Miombo woodland in Taratibu reserve, northern of Mozambique.

\begin{tabular}{|c|c|c|c|c|c|c|c|c|}
\hline Espécie / Família & $\mathrm{N}$ & FA & FR & DA & DR & DoA & DoR & IVI \\
\hline Julbernardia paniculata (Benth.) Troupin / Fabaceae & 196 & 74,2 & 5,8 & 63,2 & 17,6 & 2,7 & 16,4 & 13,3 \\
\hline Combretum adenogonium Steud. ex A.Rich. / Combretaceae & 81 & 71,0 & 5,6 & 26,1 & 7,3 & 0,9 & 5,4 & 6,1 \\
\hline Pteleopsis myrtifolia Engl. \& Diels / Combretaceae & 53 & 74,2 & 5,8 & 17,1 & 4,8 & 0,9 & 5,6 & 5,4 \\
\hline Burkea africana Hook. / Fabaceae & 61 & 71,0 & 5,6 & 19,7 & 5,5 & 0,9 & 5,2 & 5,4 \\
\hline Brachystegia spiciformis Benth. / Fabaceae & 46 & 16,1 & 1,3 & 14,8 & 4,1 & 1,8 & 10,8 & 5,4 \\
\hline Sterculia quinqueloba K.Schum. / Sterculiaceae & 63 & 41,9 & 3,3 & 20,3 & 5,7 & 1,1 & 6,4 & 5,1 \\
\hline Diplorhynchus condylocarpon (Müll.Arg.) Pichon / Ebenaceae & 63 & 64,5 & 5,1 & 20,3 & 5,7 & 0,4 & 2,5 & 4,4 \\
\hline Millettia stuhlmannii Taub. / Fabaceae & 49 & 48,4 & 3,8 & 15,8 & 4,4 & 0,9 & 5,1 & 4,4 \\
\hline Kigelia africana (Lam.) Benth / Bignoniaceae & 52 & 64,5 & 5,1 & 16,8 & 4,7 & 0,6 & 3,6 & 4,4 \\
\hline Diospyros mespiliformis Hochst. ex A.DC. / Ebenaceae & 47 & 51,6 & 4,1 & 15,2 & 4,2 & 0,8 & 4,6 & 4,3 \\
\hline Pterocarpus angolensis DC. / Fabaceae & 44 & 64,5 & 5,1 & 14,2 & 4,0 & 0,7 & 4,1 & 4,4 \\
\hline Khaya anthotheca C.DC. / Meliaceae & 40 & 38,7 & 3,0 & 12,9 & 3,6 & 0,7 & 4,0 & 3,6 \\
\hline Terminalia stenostachya Engl. \& Diels / Combretaceae & 40 & 51,6 & 4,1 & 12,9 & 3,6 & 0,4 & 2,3 & 3,3 \\
\hline Dalbergia melanoxylon Guill. \& Perr. / Fabaceae & 29 & 45,2 & 3,5 & 9,4 & 2,6 & 0,5 & 3,3 & 3,2 \\
\hline Diospyros kirkii Hiern / Ebenaceae & 33 & 54,8 & 4,3 & 10,6 & 3,0 & 0,2 & 1,4 & 2,9 \\
\hline Bauhinia galpinii N.E.Br. / Fabaceae & 30 & 48,4 & 3,8 & 9,7 & 2,7 & 0,2 & 1,3 & 2,6 \\
\hline Lannea discolor Engl. / Anacardiaceae & 26 & 54,8 & 4,3 & 8,4 & 2,3 & 0,3 & 1,6 & 2,7 \\
\hline Uapaca nitida Müll.Arg. / Euphorbiaceae & 24 & 35,5 & 2,8 & 7,7 & 2,2 & 0,3 & 1,6 & 2,2 \\
\hline Afzelia quanzensis Welw. / Fabaceae & 11 & 25,8 & 2,0 & 3,5 & 1,0 & 0,6 & 3,9 & 2,3 \\
\hline Euclea natalensis A.DC. / Ebenaceae & 18 & 32,3 & 2,5 & 5,8 & 1,6 & 0,1 & 0,5 & 1,6 \\
\hline Milicia excelsa (Welw.) C.C. Berg / Moraceae & 13 & 29,0 & 2,3 & 4,2 & 1,2 & 0,3 & 1,9 & 1,8 \\
\hline Brachystegia manga De Wild. / Fabaceae & 8 & 22,6 & 1,8 & 2,6 & 0,7 & 0,2 & 1,1 & 1,2 \\
\hline Phyllanthus pinnatus (Wight) G.L.Webster / Euphorbiaceae & 8 & 22,6 & 1,8 & 2,6 & 0,7 & 0,0 & 0,2 & 0,9 \\
\hline Baphia massaiensis Taub. / Fabaceae & 10 & 19,4 & 1,5 & 3,2 & 0,9 & 0,09 & 0,5 & 1,0 \\
\hline Thilachium africanum Lour. / Capparaceae & 8 & 9,7 & 0,8 & 2,6 & 0,7 & 0,2 & 1,3 & 0,9 \\
\hline Kigelia pinnata (Jacq.) DC. / Bignoniaceae & 7 & 19,4 & 1,5 & 2,3 & 0,6 & 0,1 & 0,4 & 0,8 \\
\hline Xeroderris stuhlmannii (Taub.) Mendonça \& E.P.Sousa / Fabaceae & 5 & 9,7 & 0,8 & 1,6 & 0,5 & 0,2 & 1,1 & 0,8 \\
\hline Artabotrys brachypetalus Benth. / Anonaceae & 4 & 9,7 & 0,8 & 1,3 & 0,4 & 0,0 & 0,3 & 0,5 \\
\hline Monotes engleri Gilg. / Dipterocarpaceae & 4 & 12,9 & 1,0 & 1,3 & 0,4 & 0,1 & 0,6 & 0,7 \\
\hline Ochna schweinfurthiana F Hoffm. / Ochnaceae & 6 & 16,1 & 1,3 & 1,9 & 0,5 & 0,0 & 0,1 & 0,6 \\
\hline Pseudolachnostylis maprouneifolia Pax / Euphorbiaceae & 5 & 16,1 & 1,3 & 1,6 & 0,5 & 0,0 & 0,2 & 0,6 \\
\hline Hymenaea verrucosa Gaertn. / Fabaceae & 5 & 12,9 & 1,0 & 1,6 & 0,5 & 0,0 & 0,2 & 0,5 \\
\hline Tamarindus indica L. / Fabaceae & 3 & 9,7 & 0,8 & 1,0 & 0,3 & 0,1 & 0,6 & 0,5 \\
\hline Sclerocarya birrea Hochst. / Anacardiaceae & 3 & 9,7 & 0,8 & 1,0 & 0,3 & 0,1 & 0,7 & 0,6 \\
\hline Brachystegia bussei Harms / Fabaceae & 3 & 6,5 & 0,5 & 1,0 & 0,3 & 0,1 & 0,5 & 0,4 \\
\hline Friesodielsia obovata (Benth.) Verdc. / Anonaceae & 5 & 6,5 & 0,5 & 1,6 & 0,5 & 0,0 & 0,2 & 0,4 \\
\hline Tabernaemontana elegans / Apocynaceae & 5 & 6,5 & 0,5 & 1,6 & 0,5 & 0,0 & 0,2 & 0,4 \\
\hline Combretum xanthothyrsum Engl. \& Diels / Combretaceae & 3 & 6,5 & 0,5 & 1,0 & 0,3 & 0,0 & 0,1 & 0,3 \\
\hline TOTAL & 1111 & - & 100 & 358 & 100 & 17 & 100 & 100 \\
\hline
\end{tabular}

Em que: $N=$ Número de árvores encontradas da espécie; $F A=$ Frequência absoluta; $F R=$ Frequência relativa (\%); $D A=$ Densidade absoluta $\left(\right.$ arv.ha $\left.{ }^{-1}\right) ; D R=$ Densidade relativa (\%); $D o A=$ Dominância absoluta $\left(\mathrm{m}^{2} . \mathrm{ha}^{-1}\right) ; D o R=$ Dominância relativa (\%); IVI = Índice de valor de importância da espécie (\%).

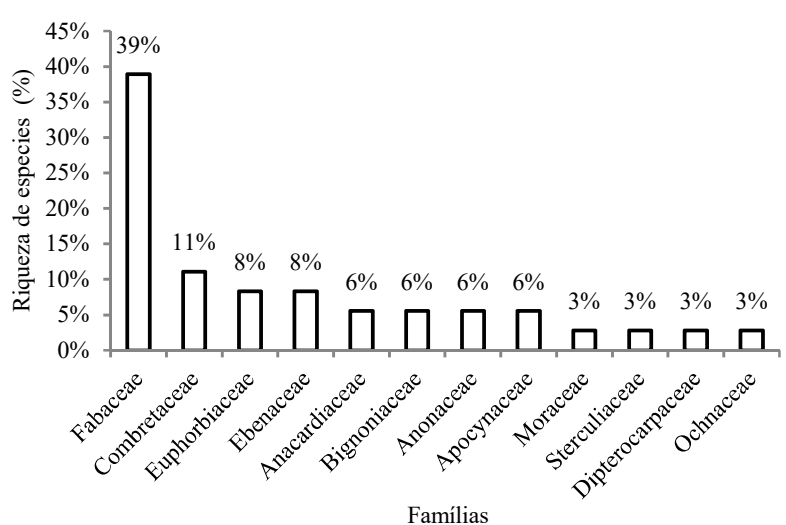

Figura 1. Principais famílias botánicas de uma floresta de Miombo da reserva de Taratibu norte de Moçambique.

Figure 1. Botanics families of the Miombo woodland in Taratibu reserve, northen of Mozambique.

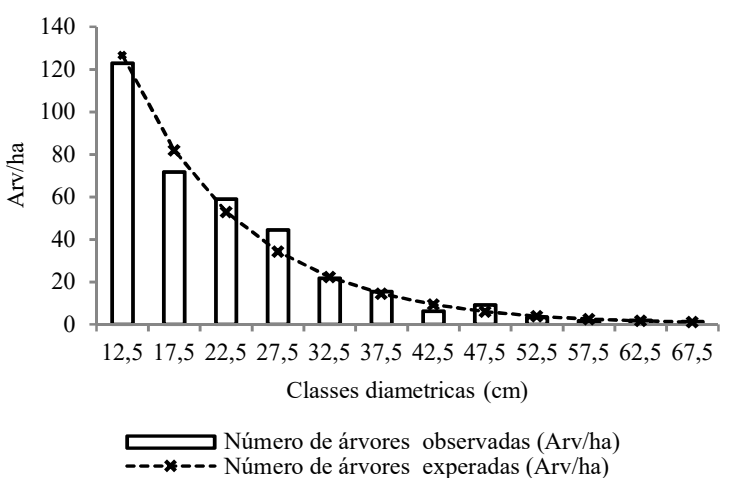

Figura 2. Número de árvores observadas e esperadas por classes de diâmetro, para a Floresta de Miombo, na reserva de Taratibu, norte de Moçambique.

Figure 2. Number of observed and estimated trees shorted by the diameter classes for Miombo woodland in the Taratibu reserve, northern of Mozambique. 


\section{DISCUSSÃO}

O número de espécies, género e famílias botânicas observadas neste estudos foram diferentes dos obtidos por Ribeiro et al (2008) em uma Floresta de Miombo na Reserva Nacional de Niassa, região norte de Moçambique, que observaram 79 espécies pertencentes a 29 famílias e uma densidade de 548 arv.ha $^{-1}$, e Hofiço et al. (2018) numa concessão florestal na região centro de Moçambique que observou 41 espécies, 12 famílias e 31 gêneros e uma densidade de 519 ind.ha $^{-1}$. Apesar disso, constatou-se semelhança na preeminência de espécies da família Fabaceae associada a família Combretaceae típico de florestas de Miombo antropizada no passado recente. Os parâmetros fitossociológicos revelaram uma comunidade caracterizada pela presença dominante de Julbernardia paniculata. Esta espécie pertence a um grupo de espécies do género Julbernardia por vezes associada a Brachystegia pertencentes a família Fabaceae que caracterizam a composição florística deste bioma (DEWEES et al. (2010). A presença marcante das espécies da família Fabaceae quando considerada como um só táxon está associada à capacidade em competir com espécies de outras famílias em solos de baixa fertilidade, que é característico deste bioma (GELDENHUYS, 2005; ISANGO, 2007; RYAN et al., 2011; KALABA et al., 2013; CHYDUMAYO, 2013). Ao passo que de acordo com Ribeiro et al. (2008) e Ryan; Williams (2011) a presença considerável de espécies da família Combretaceae nesse bioma está relacionado ao estágio sucessional da floresta, associada ocorrência de perturbações antrópica no passado, ocasionadas pela presença de fogo comumente usados na expansão de fronteiras agrícolas associado a agricultura migratória e a produção de energia a partir da biomassa florestal. Por outro lado, a presença marcante das espécies da família Euphorbiaceae pode estar relacionado a sites que são insustentáveis para a agricultura, portanto, raramente atingido pelo fogo (ZOLHO, 2005; HOFIÇO; FLEIG, 2015; GONÇALVES et al., 2017).

O número reduzido de indivíduos das espécies Millettia stuhlmannii, Pterocarpus angolensis, Dalbergia melanoxylon e Afzelia quanzensis, que estão sempre associadas as espécies dominantes deste bioma são explicados pela importância das mesmas como madeira comercial e o seu valor associado. Estas espécies são referenciadas como as espécies madeireiras mais pressionadas sob ponto de vista de corte ilegal na região feita pela comunidade local (MACKENZE, 2006; MACKENZIE; RIBEIRO, 2009; MATE et al., 2014; EIA, 2014; EKMAN et al., 2014; MITADER, 2016). Contudo, a área estudada, evidenciou alta diversidade florística, e apresentou-se homogénea na distribuição das espécies. Importa salientar que a floresta da área de estudo é caracterizada pela presença de poucas espécies dominantes, de modo que as espécies pouco comuns ou raras apresentam pequena contribuição na ocupação do espaço. Comparando o número de indivíduos com outros estudos no mesmo bioma (ISANGO, 2007; RIBEIRO et al., 2008; GILIBA et al., 2011; CHIDUMAYO, 2013; HOFIÇO; FLEIG, 2015), notou-se que a área amostrada possui menor densidade de espécimes por hectare. Porém, a baixa densidade se traduziu em alta diversidade florística, conforme observado em outros trabalhos na região (RYAN; WILLIAMS, 2011; KALABA et al., 2013). Este fato pode ter a ver com maior densidade quando uma ou mais espécies são localmente favorecidas por distúrbios antrópicos como desmatamento e degradação ocasionada por queimadas conforme relatam autores como Ryan et al. (2011) e Shirima et al. (2011), bem como ao esforço amostral empregado (KERSHAW JR et al., 2016).

O comportamento decrescente da curva de distribuição diamétrica indicou claramente a pressão antrópica sobre o ambiente florestal. A pressão antrópica pode ser constatada pelo deficit de indivíduos nas classes iniciais $(12,5 ; 17,5 \mathrm{~cm})$, e nas classes médias $(42,5 \mathrm{~cm})$. O deficit de árvores nas classes iniciais é explicada pela remoção de indivíduos menores para construção de habitação e produção de combustível lenhoso, actividade praticada comunidade local. Segundo Pereira (2006) parte da população que habita dentro dos limites deste bioma florestal, e serve-se dela como parte de estratégias de subsistência, fonte de fibra, material de construção incluindo como alternativa de alimentação durante tempos de estiagem prolongada. Já nas classes médias está relacionado à atividade de exploração ilegal madeireira comercial, facto que fora observado durante o levantamento de dados em campo.

\section{CONCLUSÕES}

A vegetação arbórea de Taratibu é caracterizada pela forte presença de espécies da família Fabaceae típica de floresta de Miombo, associadas as espécies da família Combretaceae típica de áreas com ocorrência de perturbações antrópicas. A estrutura diamétrica evidenciou uma vegetação arbórea não balanceada, e consequente necessidade de regulação da mesma.

A área evidenciou alta diversidade florística, e apresentouse homogénea na distribuição das espécies.

\section{AGRADECIMENTOS}

Ao senhor Jacobs Von Landsberg, proprietário da concessão de Taratibu pela permissão na realização da pesquisa. A Faculdade de Ciências Naturais da Universidade Lúrio-UniLúrio, pelo apoio financeiro que permitiu que a pesquisa fosse realizada.

\section{REFERÊNCIAS}

AIMI, S. C.; ARAUJO, M. M.; RORATO, D. G.; DUTRA, A. F.; CALLEGARO, R. M. Estrutura horizontal e influência de características do solo em fragmento de Floresta Ombrófila Mista. Nativa, Sinop, v. 5, n. 2, p. 151-156, 2017. DOI: http://dx.doi.org/10.5935/23187670.v05n02a12

APG THE ANGIOSPERM PHYLOGENY GROUP; CHASE, M. W.; CHRISTENHUSZ, M. J. M.; FAY, M. F.; BYNG, J. W.; JUDD, W. S.; MABBERLEY, D. J.; SENNIKOV, A. N.; SOLTIS, P. S; STEVES, P. F. The Angiosperm Phylogeny Group IV. An update of the Angiosperm Phylogeny Group Classification for the orders and families of higher plants: APG IV. Botanical Journal of the Linnean Society, v. 181, n. 1, p. 1-20, 2016. DOI: https://dx.doi.org/10.1111/boj.12385

CHIDUMAYO, E. N. Forest degradation and recovery in a Miombo woodland landscape in Zambia: 22 years of observations on permanent sample plots. Forest Ecology and Management, Amsterdam, v. 291, p. 154-161, 2013. DOI: http://dx.doi.org/10.1016/j.foreco.2012.11.031

CIENTEC CONSULTORIA E DESENVOLVIMENTO DE SISTEMAS. Software Mata Nativa 2: Sistema para Análise Fitossociológica, Elaboração de Inventários e Planos de Manejo de Florestas Nativas. Viçosa: Cientec, 2006. $295 \mathrm{p}$. 
CORAIOLA, M.; NETTO, S. P. Análise da estrutura horizontal de uma floresta estacional semidecidual localizada no Município de Cássia-MG. Revista Acadêmica: ciências agrárias e ambientais, Curitiba, v. 1, n. 4, p. 11-19. 2003. DOI: http://dx.doi.org/10.7213/cienciaanimal.v1i4.14961

DEWEES, P. A.; CAMPBELL, B. M.; KATERERE, Y.; SITOE, A.; CUNNINGHAM, A. B.; ANGELSEN, A.; WUNDER, S. Managing the Miombo woodlands of southern Africa: Policies, incentives and options for the rural poor. Natural Resources Policy Research, v. 2, n. 1, p. 57-73, 2010. http://dx.doi.org/10.1080/19390450903350846

EIA ENVIRONMENTAL INVESTIGATION AGENCY. Conexões de primeira classe: Contrabando, corte ilegal de madeira e corrupção em Moçambique.. Washington: Environmental Investigation Agency, 2014. 16 p.

EKMAN, S-M. S.; WENBIN, H.; LANGA, E. Comércio e investimento chinês na indústria madeireira de Moçambique: Um estudo de caso da província de Cabo Delgado. Bogor: CIFOR. 2014. 44 p. (Documento de trabalho, 132).

GILIBA, R. A., BOON, E. K., KAYOMBO, C. J., MUSAMBA, E. B., KASHINDYE, A. M., \& SHAYO, P. F. Species composition, richness and diversity in Miombo woodland of Bereku Forest Reserve, Tanzania. Journal of Biodiversity, v. 2, n. 1, p. 1-7, 2011.

GELDENHUYS, C. J. Basic guidelines for silvicultural and management practices in Mozambique. Report FW04/05, Forestwood, Pretoria, 2005. 78 p.

GONÇALVES, F. M. P.; REVERMANN, A. G.; AIDAR, M. P. M.; FINCKH, M.; JUERGENS, N. Tree species diversity and composition of Miombo woodlands in SouthCentral Angola: A chronosequence of forest recovery after shifting cultivation. International Journal of Forestry Research, v. 2017, p. 1-13, 2017. http://dx.doi.org/10.1155/2017/6202093

HOFIÇO, N. S. A.; FLEIG, F, D. Diversity and structure of Miombo woodlands in Mozambique using a range of sampling sizes. Journal of Agricultural Science and Technology, v. 5, n. 10, p. 679-690, 2015. https://doi.org/10.17265/2161-6264/2015.10.005.

HOFIÇO, N. S. A.; COSTA, E. A.; FLEIG, F, D.; NANVONAMUQUITXO, S. J. A. Regulation of the diametric structure of the Miombo Woodland using the De Liocourt method in Mozambique. Nativa Snop, v. 4, n. 6, p. 407-414, 2018 http://dx.doi.org/10.31413/nativa.v6i4.5396

ISANGO, J. Stand structure and tree species composition of Tanzania Miombo woodlands: A case study from Miombo woodlands of community based forest management in Iringa District. Working Papers of the Finnish Forest Research Institute, n. 50, p. 43-56, 2007.

KALABA, F. K.; QUINNA, C. H.; DOUGILL, A. J.; VINYA, R. Floristic composition, species diversity and carbon storage in charcoal and agriculture fallows and management implications in Miombo woodlands of Zambia. Forest Ecology and Management, Amsterdam, v. 304, p. 99-109, 2013. DOI: http://dx.doi.org/10.1016/j.foreco.2013.04.024

KENT, M.; COKER, P. Vegetation description and analysis, a pratical approach. Oxford: Wiley- Blackweel, 2011. $414 \mathrm{p}$.
KERSHAW JR, J. A.; DUCEY, M. J.; BEERS, T. W.; HUSCH, B. Forest Mensuration. 5. ed. Oxford: WileyBlackwell, 2016. 630 p.

MACKENZIE, C. Forest governance in Zambezia, Mozambique: Chinese takeaway! Final report for FONGZA. Maputo: Fórum das organizações não governamentais da Zambézia, 2006. 96 p.

MACKENZIE, C.; RIBEIRO, D. Tristezas tropicais: More sad stories from the forests of Zambézia. Amigos da Floresta e Justiça Ambiental, Maputo, Moçambique. 2009. $63 \mathrm{p}$.

MALMER, A. General ecological features of Miombo woodlands and considerations for utilization and management. Working Papers of the Finnish Forest Research Institute, v. 50, p. 34-42. 2007.

MATE, R.; JOHANSSON, T.; SITOE, A. Biomass equations for tropical forest tree species in Mozambique. Forests, v. 5, n. 3, p. 535-556, 2014. https://dx.doi.org/10.3390/f5030535

MILES, L.; NEWTON, A. C.; DEFRIES, R. S.; RAVILIOUS, C.; MAY, I.; BLYTH, S.; GORDON, J. E. A global overview of the conservation status of tropical dry forests. Journal of Biogeography, Oxford, v. 33, n. 3, p. 491-505, 2006. DOI: https://doi.org/10.1111/j.13652699.2005.01424.x

MAE_MINISTÉRIO D ADMINISTRAÇÃO ESTATAL. Perfil do distrito de Ancuabe, província de Cabo Delgado. 2005. Disponível em: http://www.ine.gov.mz/documentos/outrosdocumentos/perfil-do-distrito-de-ancuabe-cabodelgado.pdf/vie. Acesso em: 23/06/2018

MITADER. Defeso na exploração de pau-ferro, Swartzia madagascariensis espécie produtora de madeira de primeira classe, por período de cinco anos. Decreto Ministerial $n^{\circ}$ 10/2016. Ministério da Terra Ambiente e Desenvolvimento Rural de Moçambique (MITADER). Maputo, 2016.

PEREIRA, C. R. Estimating and mapping forest inventory variables using the K-NN method: Mocuba district case study, Mozambique. 2006. 86f. Doctoral thesis (Department of Environmental Science and Technology) Tuscia University, Viterbo, Italy, 2006.

RIBEIRO, N. S.; JETIMANE, J. L.; MILITÃO, E.; MAQUIA, I.; CHIRIZANE, C.; DE SOUSA, C.; ALVES, T.; VELOSO, M. M.; GOULAO, L. F.; RIBEIRO-BARROS, A. I. Ecological characterization of an ex situ conservation plantation in South-Eastern Mozambique. African Journal of Ecology, Oxford, v. 55, n. 1, p. 70-79, 2017. https://dx.doi.org/10.1111/aje.12320

RIBEIRO, N. S.; SHUGART, H. H.; WASHINGTONALLEN, R. The effects of fire and elephants on species composition and structure of the Niassa Reserve, Northern Mozambique. Forest Ecology and Management, Amsterdam, v. 255, n. 5, p. 16261636, 2008.2 DOI: http://dx.doi.org/10.1016/j.foreco.2007.11.033

RYAN, C. M.; WILLIAMS, M. How does fire intensity and frequency affect Miombo woodland tree populations and biomass? Ecological applications, Washington, v. 21, n. 1, p. 48-60, 2011. DOI: https://dx.doi.org/10.1890/091489.1

RYAN, C. M.; WILLIAMS, M.; GRACE, J. Above and below ground carbon stocks in a Miombo woodland landscape of 
Mozambique. Biotropica, Hoboken, v. 43, n. 4, p. 423-43.

2011. DOI: https://doi.org/10.1111/j.1744-

7429.2010.00713.x

SANTOS, R. O. dos; SOARES, R. N.; DANTAS, B. DE S.; ABREU, J. C. de; LIMA, R. B. de. Processos amostrais para estimativa de parâmetros estruturais de uma floresta estuáriana no estado do Amapá. Nativa, Sinop, v. 4, n. 5, p. 308-316. 2016. DOI: https://doi.org/10.14583/23187670.v04n05a07

SAS INSTITUTE INC. SAS Institute Inc., Cary, NC, USA. 2011.

SHIRIMA, D. D.; MUNISHI, P. K. T.; LEWIS, S. L.; BURGESS, N. D.; MARSHALL, A. R.; BALMFORD, A.; SWETNAM, R. D.; ZAHABU, E. M. Carbon storage, structure and composition of Miombo woodlands in Tanzania's eastern arc mountains. African Journal of Ecology, Oxford, v. 49, n. 3, p. 332-342. 2011. DOI: https://doi.org/10.1111/j.1365-2028.2011.01269.x

SITOE, A.; MACANDZA, V.; JORGE, P.; GABRIEL, A.; CARVALHO, M.; AMADE, F. Biodiversity baseline of the Quirimbas National Park - Mozambique. Final Report. Universidade Eduardo Mondlane. C. P. 275, Maputo, Mozambique. 2010.

VAN WYK, B.; VAN WYK, P. Field guide to trees of Southern Africa. Cape Town: Struik publishers, 2011. $536 \mathrm{p}$.

WILLIAMS, M.; RYAN, C. M.; REES, R. M.; SAMBANE, E.; FERNANDO, J.; GRACE, J. Carbon sequestration and biodiversity of re-growing Miombo woodlands in Mozambique. Forest Ecology and Management, Amsterdam, v. 254, n. 2, p. 145-155. 2008. DOI: https://doi.org/10.1016/j.foreco.2007.07.033.

WWF_WORLD WILDLIFE FUND. Só no distrito de Ancuabe: Pelo menos 89 elefantes foram mortos por furtivos nos últimos 18 meses. Mozambique: WWF, 2013. Obtido 11 de Junho de 2017, de http://www.wwf.org.mz/?1340/S-no-distrito-de-AncuabePelo-menos-89-elefantes-foram-mortos-por-furtivos-noslimos-18-meses.

ZOLHO, R. Effect of fire frequency on the regeneration of Miombo woodland in Nhambita, Mozambique. 2005. 71f. Master dissertation (Institute of Ecology and Resource Management) - University of Edinburgh, Edinburgh Scotland, 2005. 